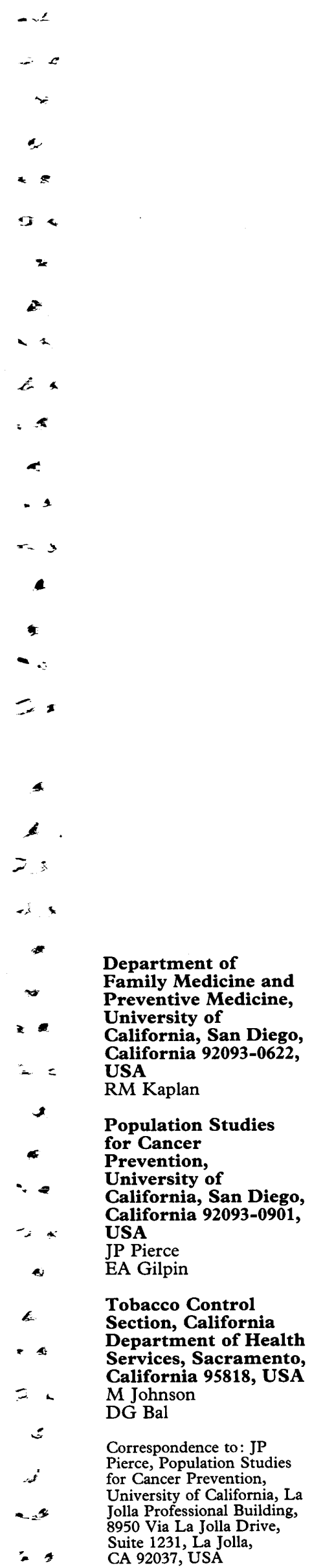

\title{
Stages of smoking cessation: the 1990 California Tobacco Survey
}

\author{
Robert M Kaplan, John P Pierce, Elizabeth A Gilpin, Michael Johnson, Dileep G Bal
}

\begin{abstract}
Objective - To estimate the distribution of smokers and former smokers among the following defined stages of the smoking cessation process: precontemplation, contemplation, preparation, cessation (or action), and maintenance.

Design - Population-based random-digit dialed telephone survey of 24296 California adults ( $\geqslant 18$ years of age) conducted in 1990. This paper focuses on 12815 smokers or former smokers who had stopped smoking in the last five years. Outcome measures-Each respondent was asked a series of questions that placed them in one of the five stages in the smoking cessation process.
\end{abstract}

Results - Among current smokers, significantly more were contemplating stopping or taking action to stop than were in the precontemplation stage. AfricanAmerican smokers were more likely to be contemplating stopping and Hispanics and African-Americans were significantly more likely to be in the preparation stages than were white nonHispanic smokers. Among those who had stopped smoking within the last 5 years, there was a trend for those with more education and of younger age to perceive themselves to be more vulnerable to relapse.

Conclusions-Public health campaigns designed to reduce cigarette smoking might benefit from targeting messages to defined demographic groups. AfricanAmerican and Hispanic smokers may be particularly receptive to cessation messages. Among those who have recently stopped smoking, younger and more educated former smokers may be particularly responsive to messages helping them to resist relapse.

(Tobacco Control 1993; 2: 139-144)

\section{Introduction}

Cigarette smokers are likely to stop smoking without having participated in a formal smoking-cessation programme; a large population-based survey in 1986 found that well over $90 \%$ of former smokers stopped by themselves. ${ }^{1}$ The same result was obtained by Schachter, ${ }^{2}$ whose retrospective interviews with convenience samples suggested that the great majority of former smokers stop smoking without external assistance. These data are also consistent with the results of other studies of smokers who try to stop smoking by themselves. ${ }^{3}$

Other evidence ${ }^{4}$ reveals smoking cessation to be a dynamic process. Over the course of time, many people alternate between smoking and non-smoking. Smoking cessation is not a discrete process but rather a complex process involving several stages. Perhaps the bestknown stage model of self-initiated smoking cessation has been proposed and refined by Prochaska, DiClemente, Velicer and colleagues. ${ }^{5-13}$ Their model divides the process into five defined stage $\left.{ }^{9}: 1\right)$ precontemplation is a stage in which people are not actively thinking about their smoking behaviour or actively considering stopping smoking; 2) during contemplation the smoker is seriously thinking about stopping smoking within the next six months; 3) smokers in the preparation stage are seriously preparing to give up cigarettes within the next month and have made at least one attempt to stop smoking in the last year; 4) the action stage is defined as the first six months after the smoker stops smoking, and 5) maintenance continues from six months after stopping smoking until the person is a confirmed non-smoker. Smokers may change from a higher to a lower stage and may move through the stages several times before eventually achieving permanent exsmoker status. The model also includes intervening constructions for self-efficacy, ${ }^{10,11}$ temptation, and decisional balance. ${ }^{12}$ Independent measures for the change process are often included. ${ }^{13}$ Various versions of the Prochaska and DiClemente model have received considerable attention, and the original model has been suggested as the basis for the design of public health interventions. However, most applications of the model have used volunteer subjects with restricted representation from various sociodemographic groups. ${ }^{10-14}$

In this paper, we apply the stages of the model to respondents in a population-based survey (the 1990 California Tobacco Survey) to examine these data for any sociodemographic differences in stages that might be useful in the design of targeted public health campaigns. 


\section{Methods}

SURVEY

The data used in this study were gathered as part of the 1990 California Tobacco Survey, a population-based, random-digit dialed telephone survey. Up to five attempts were made to reach each telephone number selected. An adult in each household identified received a screening questionnaire to enumerate all household members and to collect basic demographic information for each, including smoking status information for persons 10 years of age or older. Of attempts to contact telephone numbers identified as households, $75.1 \%$ resulted in completed screening questionnaires. Next, in-depth questionnaires were administered to all persons in the household who currently smoked or had stopped smoking in the past 5 years (Adult Questionnaire). Non-smokers and those who had stopped smoking more than 5 years before, were sampled with a probability of 0.28 . Completed interviews were obtained for $75.3 \%$ of those selected for the extended questionnaires.

All interviews were conducted using a computer-assisted telephone interview (CATI) system. Respondents who preferred to be interviewed in Spanish were given a Spanish-speaking interviewer and a Spanish version of the questionnaire. After administration of the screening questionnaire, the CATI system automatically selected the household members who would be asked to complete the extended questionnaires. The adult interview took about 15 minutes. Details of the sampling methods concerning telephone number selection, clustering, weighting, and precision of estimates can be obtained from the authors. A full description of the California Tobacco Survey has been published as a technical report. ${ }^{15}$

\section{SAMPLE}

Overall, the adult questionnaire was administered to 24296 individuals 18 years of age and older. This paper focuses on the subset of 12815 adult smokers and ex-smokers who had stopped smoking in the last 5 years. The demographic composition of the study group and sample sizes are shown in the table by smoking status at the time of the survey.

Current smokers were asked the following questions: 1) "During the past 12 months, have you quit smoking intentionally for 1 day or longer?" 2) "Are you planning to quit in the next month?" and 3) "If not, are you contemplating quitting in the next 6 months?" Their answers were used to categorise the respondents into discrete groups that represent the latest refinement of the stages of quitting model (WF Velicer and JO Prochaska, personal communication). Respondents answering "no" to all three questions were considered to be in the precontemplation stage. Those who answered "yes" to questions 1 and 2 were considered to be in the preparation stage. Other smokers who answered "yes" to either question 2 or 3 were considered to be in the contemplation stage. Former smokers were
Table Demographic characteristics and sample sizes for current and former smokers $(n=12815)$

\begin{tabular}{lccc}
\hline & \multicolumn{2}{c}{$\begin{array}{c}\text { Smoking status at } \\
\text { time of survey }\end{array}$} & \\
\cline { 2 - 3 } & Current & Former & $\begin{array}{c}\text { Sample } \\
\text { size }\end{array}$ \\
\cline { 2 - 3 } & 9534 & 3281 & \\
$\mathrm{n}$ & $73.5 \pm 1.3$ & $26.6 \pm 1.3$ & 100.0 \\
Weighted \% & & & \\
Sex & $74.0 \pm 1.7$ & $26.0 \pm 1.7$ & 6313 \\
Male & $72.7 \pm 1.8$ & $27.3 \pm 1.8$ & 6502 \\
Female & & & \\
Age (years) & $74.5 \pm 3.9$ & $25.5 \pm 3.9$ & 1825 \\
18-24 & $75.9 \pm 1.6$ & $24.1 \pm 1.6$ & 6439 \\
25-44 & $71.8 \pm 2.6$ & $28.2 \pm 2.6$ & 3558 \\
45-64 & $61.1 \pm 4.1$ & $38.9 \pm 4.1$ & 993 \\
65+ & & & \\
Race/ethnic group & $71.8 \pm 3.7$ & $28.2 \pm 3.7$ & 1532 \\
Hispanic & $73.1 \pm 1.4$ & $26.9 \pm 1.4$ & 9796 \\
White (non-Hispanic) & $77.9 \pm 6.5$ & $22.1 \pm 6.5$ & 704 \\
African-American & $71.8 \pm 6.3$ & $28.2 \pm 6.3$ & 520 \\
Asian/Pacific Islanders & $84.7 \pm 5.6$ & $15.3 \pm 5.6$ & 263 \\
Other & & & \\
Education (years) & $76.8 \pm 3.0$ & $23.2 \pm 3.0$ & 1734 \\
<12 & $75.1 \pm 2.0$ & $24.9 \pm 2.0$ & 4666 \\
12 & $71.9 \pm 2.1$ & $28.1 \pm 2.1$ & 4184 \\
13-15 & $65.4 \pm 3.7$ & $34.6 \pm 3.7$ & 2231 \\
16+ & & & \\
\hline
\end{tabular}

Numbers are percentages $\pm 95 \%$ confidence intervals. NB $95 \%$ confidence intervals are the same for current and former smokers since together they define the study population.

classified as being in the action stage if they had been abstinent for less than 6 months, and in the maintenance stage if abstinence had lasted from between 6 months and 5 years. Former smokers were also asked, "Do you think that it is likely or unlikely that you will return to smoking in the next 12 months?"; those answering "likely" were considered to be vulnerable to relapse.

\section{STATISTICS}

Percentages were obtained for the various categories broken down by demographic groups, and $95 \%$ confidence intervals were computed using a variant of the jackknife procedure, ${ }^{16}$ in which 33 subsamples were taken from the full survey file, and sample weights were computed according to the same procedure as for the full sample. Variances were estimated on the basis of the deviations of the subsample percentages and the percentage for the full sample. The variances were then used to compute the $95 \%$ confidence intervals in the usual manner on the basis of the critical value from the Student's " $t$ " distribution. Also, chi-square statistics were used to examine the distribution of demographic subgroups according to stage; they were computed using a method (Satterthwaite's approximation) based on the subsamples (see above) and adjusted for the survey design. ${ }^{17,18} \mathrm{Chi}$-square statistics were also computed to examine the distribution of demographic variables within the action and maintenance groups for those former smokers who considered themselves vulnerable to relapse. These were one degree of freedom tests. The likelihood of being in a given stage was modeled using logistic regression with simultaneous entry of the demographic predictor variables. ${ }^{19}$ Again, a jackknife method was used to compute $95 \%$ confidence intervals for the risk ratios (rr) derived from the regression coefficients. 


\section{Results}

STAGES OF QUITTING

The distributions of stages of quitting for men and women are shown in figure 1. Gender differences were non-significant (by the chisquare test), as demonstrated by the overlapping $95 \%$ confidence intervals. Overall, $27.4 \%$ of the respondents were in the precontemplation stage, $34.3 \%$ in the contemplation stage, $11.8 \%$ in the preparation stage, $5.9 \%$ in the action stage, and $20.7 \%$ in maintenance. When age, race/ethnic group, and education were examined with respect to stages of quitting, the stage distribution differed for the various levels of each factor (all $\mathrm{p}<0.001)$. The stages for current and former smokers are described separately below.

\section{Current smoker stages}

Figure 2 (top) shows the distribution of respondents in the first three stages (precontemplation, contemplation, and preparation) by race/ethnic group. Although differ-

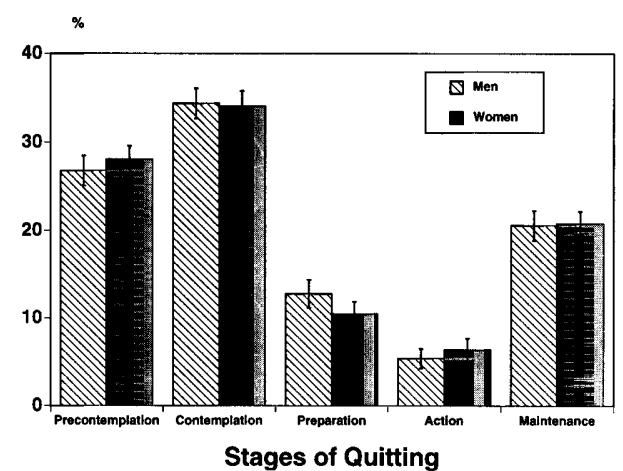

Figure 1 Distribution of stages of quitting for current and recent former smokers by gender.
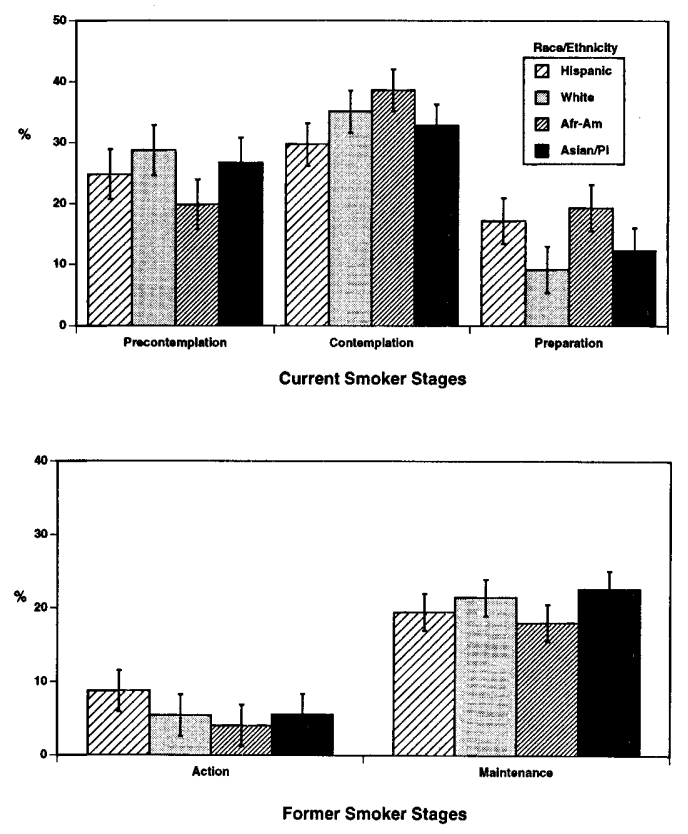

Figure 2 Top: current smoker stages by race/ethnic group; bottom: former smoker stages by race/ethnic group. ences between groups were small and the $95 \%$ confidence intervals overlapped, the percentage contemplating stopping smoking was highest among African-Americans (38.6\%). A significantly higher percentage of Hispanics $(17.2 \%)$ and African-Americans $(19.4 \%)$ than whites $(9.2 \%)$ were in the preparation phase; the percentages of Hispanics and AfricanAmericans in this phase were higher than that of Asians/Pacific Islanders $(12.3 \%)$, but these differences were not significant.

The percentage in the contemplation stage varied by age (figure 3 , top). The percentage of those between 25 and 44 years of age in the contemplation stage $(37.8 \%)$ was higher than the percentage of those 45-64 years old $(30.7 \%)$ and much higher than the percentage of those 65 years of age or older $(20.8 \%)$ (figure 3, top). However, with respect to educational level, the only trends found were related to the precontemplation and preparation stages; although the $95 \%$ confidence intervals overlapped, a slight trend was seen toward smaller percentages in the precontemplation and preparation stages with higher educational level (figure 4 , top).

\section{Former smoker stages}

For respondents who were abstinent at the time of the survey, no marked differences were found in the percentages in the action and maintenance stages by race (figure 2 , bottom), although African-Americans had a slightly lower percentage $(18.1 \%)$ in maintenance than did the other groups. However, differences were found with age (figure 3 , bottom) and education (figure 4 , bottom). The percentage in the action stage decreased slightly with age; the percentage in maintenance increased significantly with age, doubling between the
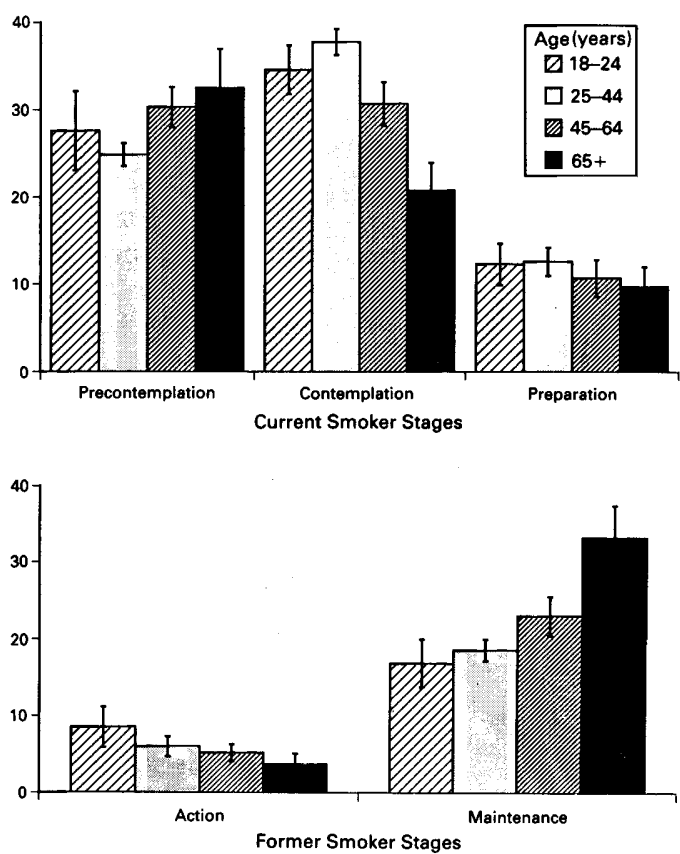

Figure 3 Top: current smoker stages by age; bottom : former smoker stages by age. 

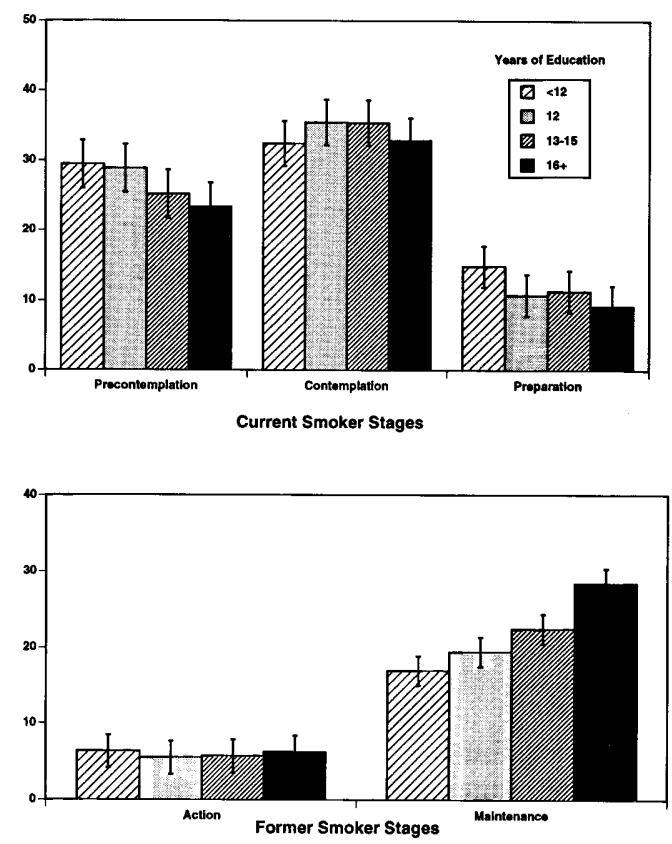

Figure 4 Top: current smoker stages by education; bottom: former smoker stages by education.

18-24 group (16.9\%) and the group aged 65 or older $(32.9 \%)$. Among the education groups, the percentages in the action stage were similar in all groups, but a clear trend was seen toward an increased percentage in maintenance with more years of formal education.

\section{VULNERABILITY TO RELAPSE}

To determine whether the distribution of quitters vulnerable to relapse differed by demographic group, the percentage of respondents who were in the action or maintenance stages $(n=3281)$ but said they were likely to be smoking again in 12 months was plotted against the time elapsed since these former smokers had stopped smoking. For both men and women, vulnerability was highest in the action stage and decreased only slightly (if at all) in the early maintenance stage. By the second year, vulnerability was reduced significantly for men; although it was also reduced for women, the differences are only significant when 2-year vulnerability is compared to vulnerability in the action stage (figure 5).

When those who considered themselves vulnerable to relapse were examined further to determine whether the distribution of gender, age, race/ethnicity, and education groups differed over the action and maintenance groups, stage distribution only differed according to age $(\mathrm{p}<0.02)$

\section{MULTIVARIATE ANALYSIS}

Two multivariate analyses were performed. First, logistic regression was used to model the likelihood of being in the preparation stage versus in the contemplation or precontemplation stages. After adjustment for the other demographic factors, Hispanics showed an

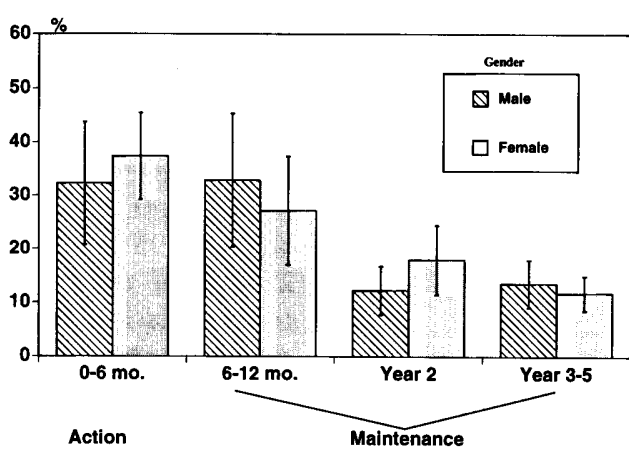

Figure 5 Percentage of former smokers who consider themselves vulnerable to relapse by gender.

increased likelihood of being in the preparation stage, $\mathrm{rr}=2.07(95 \% \mathrm{CI}: 1.48-2.89)$ compared to whites, as did African-Americans, $\mathrm{rr}=2.30(95 \% \mathrm{CI}: 1.61-3.29)$. None of the other demographic factors reached statistical significance.

For those who had stopped smoking, a logistic regression was performed to model the likelihood of being in the maintenance versus action stages. After adjustment for the other demographic factors, age 45-64 years ( $\mathrm{rr}=$ $2.05,95 \% \mathrm{CI}: 1.36-3.09)$ and age 65 or more years ( $\mathrm{rr}=4.07,95 \% \mathrm{CI}: 2.36-7.02$ ) had an increased likelihood of being in the maintenance stage compared to the age group 18-24 years. None of the other demographic factors was statistically significant.

\section{Discussion}

Although cigarette use is declining in California, ${ }^{20}$ more than $20 \%$ of the adult population still smokes. In 1988, the California Legislature enacted Proposition 99, which increased the cigarette tax by $\$ 0.25$ per pack. A large portion of revenue from that tax finances mass-media and public-education campaigns to reduce tobacco use. A theoretical model developed and modified by Prochaska, DiClemente and colleagues ${ }^{5-13}$ suggested that the process of eliminating smoking behaviour is characterised by discrete stages. Previous studies have not identified the distribution of these stages in the general population, nor have they characterised the distribution among sociodemographic groups. Knowledge of the stage profile in different groups might help public health officials to design messages and programmes targeted more directly toward the different groups.

Giving up cigarette smoking is a difficult task. The natural course of smoking cessation typically involves multiple attempts. It is not uncommon for a person to stop smoking, begin again, and to go through multiple cycles of smoking, smoking cessation, and varied periods of abstinence. ${ }^{7,8}$ The Transtheoretical model and other intention theories may provide significant insight into this cyclic process because they link the cessation process to specific time intervals. However, the insight given by cross-sectional studies is limited. Future longitudinal studies are required in 
order to understand the cessation process among California citizens.

Our results suggest few differences between men and women with regard to their distribution in these stages, but women may feel vulnerable to relapse for a somewhat longer period than men. Other studies have presented evidence that women have a more difficult time stopping smoking than do men. ${ }^{20,21}$

African-Americans were more likely than those in other racial/ethnic groups to be contemplating stopping smoking, but the percentage in the maintenance stage was less than the percentage in other groups. Thus, it might be important to help this group progress to action and support them throughout the action phase so that they reach maintenance. AfricanAmericans who had stopped smoking tended to consider themselves less vulnerable to relapse than other groups, so a different approach with respect to support may be appropriate. The multivariate analysis demonstrated that, after adjustment for other variables, being Hispanic was the strongest predictor of being in the preparation stage. Thus, Hispanics may be more likely to be thinking about stopping smoking within the next month and the distribution of culturally relevant materials to assist this effort may be profitable. Clearly, more demographic analysis is needed. For example, our results appear to conflict with a worksite study demonstrating that low socioeconomic status workers are the least likely to be contemplating a quit attempt. ${ }^{22}$

Although the sample size for this study is large, generalisations must be limited to California. California is unusual among US states for several reasons. First, California has a relatively low smoking prevalence at about $21 \%$ (the national average excluding California is closer to $27 \%$ ). ${ }^{8}$ In addition, the demographic characteristics of California are unique, with a significantly higher proportion of Hispanics than most other states. The closest national sample that provides data on stages is the Adult Use of Tobacco Survey. ${ }^{23} \mathrm{~A}$ 12-month follow-up interview of subjects in that study revealed a distribution among stages similar to this study. Identical numbers were in the contemplation stage while the California sample had fewer people in the precontemplation stage $(27 \%$ versus $35 \%$ ), approximately the same number in the contemplation stage $(12 \%$ versus $15 \%$ ), and more people in the action and maintenance stages $(27 \%$ versus $16 \%$ ). The observation that there are more people in the action and maintenance stages is consistent with reports that more Californians have been successful in stopping smoking than residents of other states have been.

Gritz and colleagues ${ }^{24}$ recently reported data on stages of change for a group of 1396 female members of a health maintenance organisation (HMO). Data from this study are not exactly comparable with the present study because their sample only included current smokers. Thus, at baseline there were no individuals in the maintenance stage. In order to make our figures comparable to those reported by Gritz et al, we recalculated the percentages by adjusting the denominator to exclude those in the maintenance stage. The California population sample had slightly more people in the precontemplation stage (34\% versus $30 \%$ ) and significantly fewer in the contemplation stage $(43 \%$ versus $58 \%$ ). The HMO study identified $8 \%$ in the preparation stage in contrast to $15 \%$ in the California study. There were $4 \%$ in the action stage among enrollees in HMOs as opposed to $7 \%$ in the California study. It is worth noting that a substantial proportion of California citizens are medically uninsured, with about one-sixth of the state in the MediCal (California's Medicaid Programme) system. Enrollees in HMOs tend to be more affluent and more educated. The discrepancies between the distributions of the stages in the Gritz study and the corresponding California Tobacco Survey cells for welleducated females are relatively small. Overall, there is reasonable correspondence between the population-based data and those obtained with convenience samples.

These results must be interpreted cautiously for several reasons. First, although the sample size for the California Tobacco Survey is large, the number of respondents in some unique cells of the analysis is small. For example, there were relatively few black female contemplators. There is also some evidence that biases from telephone interviewing methods are greater for some ethnic or racial groups than for others. ${ }^{25,26}$ We hope that our results will be confirmed by surveys using personal interviews. It is also important to emphasise that our measure of vulnerability to relapse is rather crude. The self-report measure does not allow a good assessment of abstinence. Validation of self-reports in future studies will be important for several reasons. For example, the finding that higher educated subjects and women may be more vulnerable to relapse could, potentially, be an artifact, as it is possible that these groups were more willing to report such vulnerability. Thus, the results could represent reporting bias rather than actual vulnerability.

Older smokers are less likely to contemplate stopping smoking; however, the percentage of smokers in the population systematically declines with age. Thus, the older group may represent "hardened" smokers (the ones remaining after most others in their birth cohort have died or successfully stopped smoking), and targeting programmes to younger smokers might produce more benefits than targeting programmes to older groups. Also, older persons in the action and maintenance stages considered themselves less vulnerable to relapse. The multivariate result showing that advanced age is a predictor of being in the maintenance stage was expected, because the analysis includes former smokers who stopped smoking up to five years ago. For this group there is also a bias because having lived longer provides more years to have been a former smoker. Several other studies also confirm that success in stopping smoking is related to age. ${ }^{8}$ On the other hand, it is worth noting that, in the analysis of a national survey of the American Association of Retired Persons by 
Orleans and colleagues, among 289 smokers aged 50 to 74 years, most reported some interest in stopping smoking. ${ }^{27}$

In summary, smokers and recent former smokers represent various stages along the smoking continuum, from those not contemplating stopping smoking to those approaching true termination of the behaviour. The distribution among these stages is related to sociodemographic variables, including race, age, and education. Also, vulnerability to relapse is related to age and may be related to the other demographic factors as well among those in the action and maintenance stages. Public health campaigns designed to reduce cigarette smoking must recognise that people differ in their readiness to quit and in their vulnerability to relapse. Further, the distribution within these categories differs by some demographic characteristics.

This work was supported by a contract to John P Pierce from the California Department of Health Services by the UCSD Cancer Center Foundation and by Cancer Center Gran P30 CA 23100 from the National Cancer Institute.

1 Fiore MC, Novotny TE, Pierce JP, et al. Methods used to quit smoking in the United States: do cessation programs quit smoking in the United States

2 Schachter S. Recidivism of self-cure of smoking and obesity. Am Psychol 1982; 37: 426-44.

3 Cohen S, Lichtenstein E, Prochaska JO, et al. Debunking myths about self-quitting. Evidence from 10 prospective studies of persons who attempt to quit smoking by themselves. Am Psychol 1989; 44: 1355-65.

4 Swartz JL. Review and Evaluation of Smoking Cessation Methods: United States and Canada, 1978-1985. National Institutes of Health Publication No. 87-2940. Bethesda, MD: US Department of Health and Human Services, 1987.

5 Prochaska JO, Diclemente CC. Stages and processes of selfchange of smoking: toward an integrated model of change of smoking: toward an integrated mode

6 Prochaska JO, Diclemente CC, Velicer WF, Ginpil S, Norcross JC. Predicting change in smoking status for

7 Velicer WF, Prochaska JO, Rossi JS, Snow MC. Assessing outcome in smoking cessation studies. Psychol Bull 1992; 111: 23-41.

8 US Department of Health and Human Services. Reducing the health consequences of smoking: 25 years of progress. $A$ report of the Surgeon General, 1989. Atlanta, Georgia: Centers for Disease Control, Office on Smoking and Health, 1989. (DHHS Publication no (CDC) 89-8411.)
9 DiClemente CC, Prochaska JO, Fairhurst SK, Velicer WF, Valesquez MM, Rossi JW. The processes of smoking cessation: an analysis of precontemplation, contemplation, and preparation stages of change. $\mathcal{F}$ Consult Clin plation, and preparation stages

10 DiClemente CC, Prochaska JO, Gilbertini M. Self-efficacy in the stages of self-change of smoking. Cognitive Ther in the stages of self-ch

11 Velicer WF, DiClemente CC, Rossi JS, Prochaska JO. Relapse situations and self-efficacy: an integrative model Addict Behav 1990; 15: 281-93.

12 Velicer WF, DiClemente CC, Prochaska JO, Brandenberg N. Decisional balance measure for assessing and predicting smoking status. $\mathcal{F}$ Pers Soc Psychol 1985; 48 1279-89.

13 Prochaska JO, Velicer WF, DiClemente CC, Fava J. Measuring the processes of change: applications to the cessation of smoking. $\mathcal{f}$ Consult Clin Psychol 1988; 56 : $520-8$.

14 Wilcox MS, Prochaska JO, Velicer WF, DiClemente CC. Subject characteristics as predictors of self-change in Subject characteristics as predictors of

15 Burns D, Pierce JP. Tobacco use in California 1990-1991. Sacramento: California Department of Health Services, 1992.

16 Efron B. The jackknife, the bootstrap and other resampling plans. Philadelphia, PA: Society for Industrial and Applied Mathematics, 1982.

17 Rao JNK, Scott AJ. The analysis of categorical data from complex sample surveys: chi-squared tests for goodness of fit and independence in two-way tables. $7 \mathrm{Am}$ Stat Assoc 1981; 76: 221-30.

18 Rao JNK, Scott AJ. On chi-squared tests for multiway contingency tables with cell proportions estimated from survey data. Ann Stat 1984;12:46-60.

19 SAS Technical Report P-200. Release 6.04. Cary NC: The SAS Institute, 1990: 175-230.

20 Pierce JP, Burns DM, Berry B, Goodman J, Gilpin E. Reducing tobacco consumption in California: ProposiReducing tobacco consumption in California: Proposi
tion 99 seems to work. $\mathcal{7} A M A 1991 ; 265: 1257-8$.

21 Pierce JP, Fiore MC Novotny TE, Hatiandrew EJ, Davi RM. Trends in cigarette smoking in the United States. Educational differences are increasing. $\mathcal{F} A M A 1989 ; 261$ 56-60.

22 Abrams DB, Biener L. Motivational characteristics of smokers at the workplace: a public health challenge. Prev Med 1992; 21(6): 679-87.

23 Prochaska JO. What causes people to change from unhealthy to health enhancing behaviour? In: Cummings CC, Floyd JD, eds. Human behavior and cancer risk reduction: overview and report of a conference on unmet reduction: overview and report of a conference on unmet

24 Gritz ER, Berman BA, Bastani R, Wu M. A randomized trial of a self-help smoking cessation intervention in a
nonvolunteer female population: testing the limits of the nonvolunteer female population: testing the limits of the

25 Aquilino WS, LoSciato LA. Effects of interview made on self-reported drug use. Public Opinion $Q$ 1990; 54 362-95.

26 Bachman JG, O'Malley PM. Yea-saying, nay-saying, and going to extremes: are black-white differences in survey results due to response style? Public Opinion $Q$ 1984; 48 : 409-27.

27 Orleans CT, Rimer BK, Cristinzio S, Keintz MK, Fleisher L. A national survey of older smokers: treatment needs of a growing population. Health Psychol 1991; 10(5). 343-51.

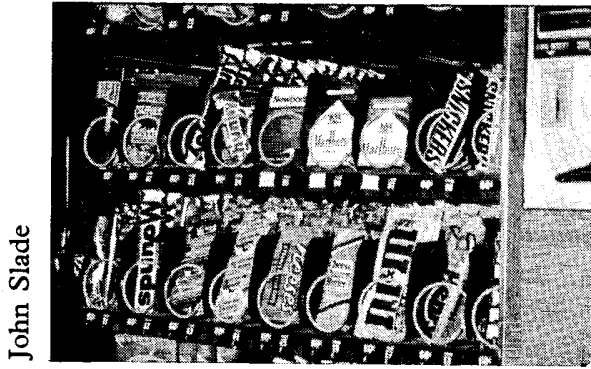

Candy and cigarette vending machine (New

fersey Statehouse, Trenton, NF, USA) 